\title{
A Cross-Layered Approach for Improving Quality of Service of Video Transmission in Ad-hoc Networks
}

\author{
Mohammed Yasin Ali \\ Research Scholar, Department of Electronics and Communication Mewar University \\ Chittorgarh, Rajasthan, India \\ yaseencms@gmail.com \\ Syed Musthak Ahmed \\ Dean (Academics), Department of Electronics and Communication S. R. Engineering College \\ Warangal, Telangana, India \\ syedmusthak_gce@yahoo.com
}

\begin{abstract}
Over the last years a number of new protocols have been developed for multimedia applications in the whole OSI layer's scale. In addition wireless communications and networking fast occupy centre stage in research and development activity in the area of communication networks. In order to support better wireless user the cross layer design paradigm has been proposed. This chapter presents the challenges in design and implementation of cross layer adaptation schemes for multimedia transmission over wireless networks. In addition this chapter presents the most important parameters and constrains that should be taken into consideration when attempting cross layer adaptation in wireless networks that involves different protocols in the overall protocol stack.
\end{abstract}

Keywords: WSN, Cross Layer, Video Transmission

Received: 24 December 2017, Revised 4 February 2018, Accepted 17 February 2018

(C) 2018 DLINE. All Rights Reserved

\section{Introduction}

Adhoc networks are a key factor in the evolution of wireless communications. Self-organized ad hoc networks of PDAs or laptops are used in disaster relief, conference, and battlefield environments. These networks inherit the traditional problems of wireless and mobile communications, such as bandwidth optimization, power control, and transmission-quality enhancement. In addition, their multihop nature and the possible lack of a fixed infrastructure introduce new research problems such as network configuration, device discovery, and topology maintenance, as well as ad hoc addressing and self-routing.

In ad hoc networks, wireless hosts can communicate with each other in the absence of a fixed infrastructure. These networks typically consist of equal nodes that communicate over wireless links without central control.

$48 \quad$ Journal of Networking Technology Volume 9 Number 2 June 2018


Multihop cellular networks have recently emerged as a communication alternative at events where huge numbers of users are concentrated in a small area such as a stadium. Peer-to-peer networks are ad hoc networks in which an overlay network is built on the Internet. In a P2P network, two or more peers can use appropriate information and communication systems to collaborate spontaneously without requiring central coordination.

Some ad hoc networks are linked to a fixed infrastructure via access points. For example, mesh or rooftop networks (www.sonic.net/ sales/rooftop/ faq.shtml) consist of antennas placed on top of buildings to provide wireless Internet access. Vehicles on a highway can create an ad hoc network for use in disseminating traffic information. They can operate as a pure ad hoc network in which an individual vehicle detects traffic events and initiates a broadcast to other vehicles. Alternatively, cellular or Internet access points placed near the road can transmit the information. Multihop cellular networks3 have recently emerged as a communication alternative at events where huge numbers of users are concentrated in a small area such as a stadium. Peer-topeer networks are ad hoc networks in which an overlay network is built on the Internet. In a P2P network, two or more peers can use appropriate information and communication systems to collaborate spontaneously without requiring central coordination.

Cross-layer optimization is an escape from the pure waterfall-like concept of the OSI communications model with virtually strict boundaries between layers. The cross-layer approach transports feedback dynamically via the layer boundaries to enable the compensation for e.g. overload, latency or other mismatch of requirements and resources by any control input to another layer but that layer directly affected by the detected deficiency.

In the original OSI networking model, strict boundaries between layers are enforced, where data are kept strictly within a given layer. Cross layer optimization removes such strict boundaries to allow communication between layers by permitting one layer to access the data of another layer to exchange information and enable interaction. For example, having knowledge of the current physical state will help a channel allocation scheme or automatic repeat request (ARQ) strategy at the MAC layer in optimizing tradeoffs and achieving throughput maximization.

Especially in information routing with concurrent demand for limited capacity of channels there may be a need for a concept of intervention to balance between e.g. the needs of intelligible speech transmission and of sufficiently dynamic control commands. Any fixed allocation of resources will lead to a mismatch under special conditions of operations. Any highly dynamic change of resource allocation might affect the intelligibility of voice or the steadiness of videos. However, as with other optimizing strategies, the algorithm consumes time as well.

Cross-layer optimization shall contribute to an improvement of quality of services under various operational conditions. Such adaptive quality of service management is currently subject of various patent applications. The cross-layer control mechanism provides a feedback on concurrent quality information for the adaptive setting of control parameters. The control scheme apply.

- The observed quality parameters.

- A fuzzy logic based reasoning about applying the appropriate control strategy.

- The statistically computed control input to parameter settings and mode switches.

\section{Literature Survey}

So many research works done in this area. Rate adaption is performed under two categories. Those are static based and channel quality based approach. In static based approach using frame error or bit error we can achieve necessary information on wireless channel for rate adaption. In channel quality based approach estimate the channel quality based on the measured SNR and adjust the data transmission mode by predefined threshold values. Channel quality based approach has three techniques. Those are sender based, received based and hybrid adaption techniques. Several rate adaption algorithms have been proposed over the past decades. Those algorithms focus on adjust their transmission rate with the help of channel feedback from mac layer. Most widely used rate adaption algorithm is (ARF) auto rate fall back. ARF algorithm is like a trial and error approach to select the bit rate which is optimal. If the channel is fluctuating rapidly this algorithm is not suitable for those types of channels.

Link failure management schemes based on physical layer parameters to identify the link quality. Signal strength is used to find the link quality at network layer. In proposed cross-layer uses link residual time (LRT) as a metric to identify link quality based 
on received power absorbed at the physical layer. Link residual time value is used in upper layers to take better routing decisions for sending packets.

Mobile Ad hoc network has limited resources so energy efficiency is more important to handle. Many algorithms have proposed for handling resources in efficient manner [15]. Energy efficient routing in MANETs divided into two categories. Transmission control approach and load distribution approach. In transmission control approach energy efficient routing is based on find the best route that minimizes the total transmission power [15]. In load balancing algorithms are there to improve the efficiency of the system by transferring packets from overloaded to other unloaded nodes.

\section{Related Work}

Cross layer adaptation is a very challenging process due to the numerous parameters involved in the whole procedure. This section outlines the most important parameters and constrains that should be taken into consideration when attempting cross layer adaptation in wireless networks that involves different protocols in the overall protocol stack.

In multimedia transmission three entities can be distinguished that take part in the information exchange procedure: the sender, the core network elements (links, routers) and the receiver. The term sender includes either a multimedia server or an individual host which participates in a multimedia data exchange with another remote host. There has been a detailed discussion whether or not all three elements should be involved in an adaptation scheme, targeting at improving the QoS offered to the end user. The most challenging and maybe the most beneficial approach would be the participation of all three elements in the adaptation mechanism especially when the multimedia data are transmitted among various network domains. However, even in the same network domain someone has to decide whether or not both the sender and the receiver should participate in the adaptation process. Someone should also consider that the complexity increases when inter-domain adaptation and policies are to be implemented. Therefore, the cross-layer adaptation scheme that is related to the participation of the entities involved in the multimedia transmission can fall into the next four categories:

- Sender Based: The sender performs the cross-layer adaptation. This approach has the advantage of easy deployment due to the fact that it does not require any support from the network or the receivers. On the other hand, this approach has limited capabilities.

- Receiver Based: The receiver performs the cross-layer adaptation. This approach also has the advantage of easy deployment due to the fact that it does not require any support from the network or the sender. Again, this approach has limited capabilities.

- Network Supported: The network elements are involved in the cross-layer adaptation. In a heterogeneous environment, such as the Internet, agreements have to be set up amongst the various network domains to ensure any cross layered implementation across the path between the sender and the receiver. In the same domain, the administrator cab define their own policiesmechanisms.

- Hybrid: A combination of two or more of the above approaches. This approach is the most complicated to be implemented but has the potential to provide better performance.

\section{Proposed Work}

The conceptual design of the research methodology is illustrated in Figure 1. It provides the activities involved in the research.

As can be seen in Figure 1, the outline of the research is provided. First of all, the present state of the art on crosses layered approach for optimizing media transmission in wireless networks. It provides useful insights that can be compared with our research results. Then a framework is proposed and implemented that can help in selecting strategies of different layers and jointly optimize in order to improve performance. Then the proposed approach is evaluated and compared with prior approaches. Afterwards conclusions are drawn and recommendations are provided. Figure 2 shows more technical details of the proposed solution.

As can be seen in Figure 2, the cross layered approach is explored in both transmission and receiving of medical video. The encoding and decoding are used as per the requirements for video transmission efficiently. The error concealment is the phase

\begin{tabular}{llllll}
\hline 50 & Journal of Networking Technology Volume 9 & Number 2 & June 2018 \\
\hline
\end{tabular}




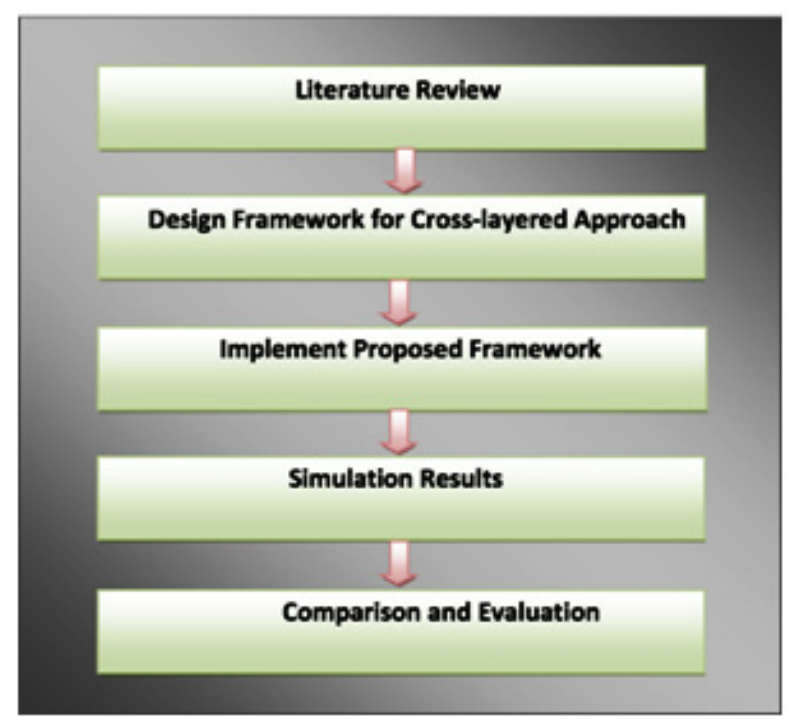

Figure 1. Illustrates the proposed methodology

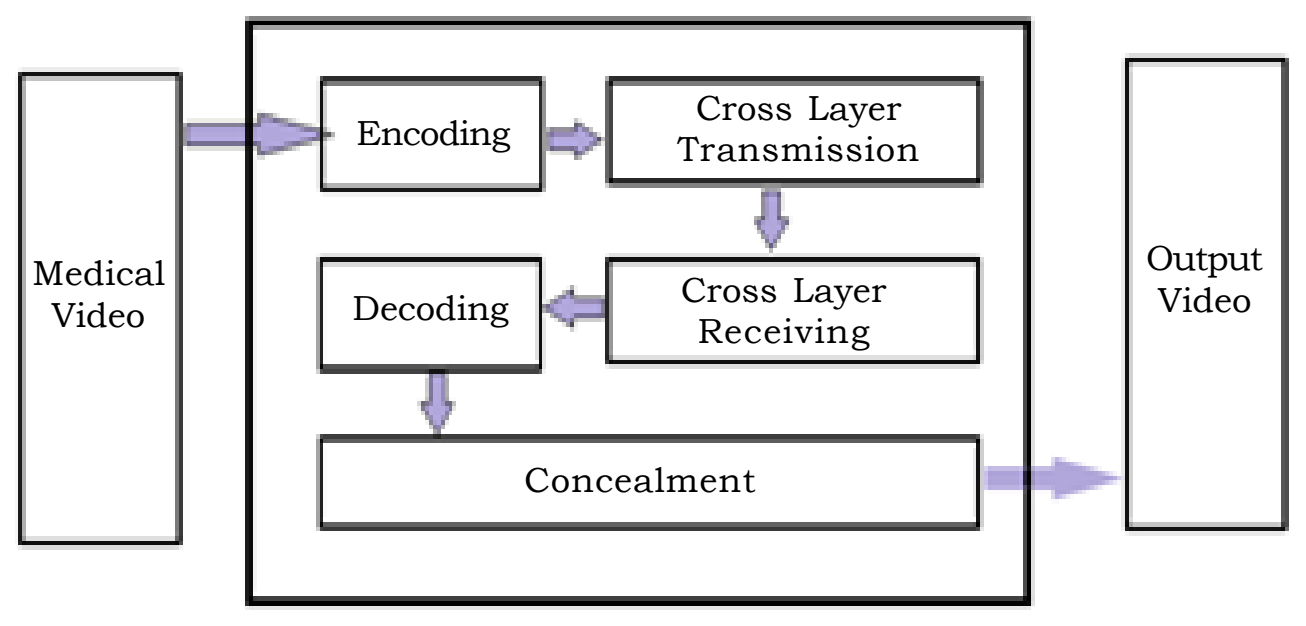

Figure 2. Proposed solution

in which the lost packet are replaced and the quality of the video transmission is improved.

\section{Problem Statement and Assumptions}

Another important issue is how the adaptation strategy could be realized (Xiuyuan, 2008). There are various approaches in this field as following:

- Bottom-up Approach: In this approach the lower layers (PHY and MAC) provide the upper layers with optimal services by reducing the transmission errors (not efficient for multimedia transmission).

- Top-down Approach: In this approach the APP layer informs the lower layers for the importance of each data packet and the lower layers treat each data set with a different way, based on QoS criteria. The higher layer protocols optimize the parameters and the strategies of the next lower layer.

- MAC-centric Approach: In this approach the APP layer passes its traffic information and requirements to the MAC layer that 
decides which APP layer packets/flows should be transmitted and at what QoS level.

- Integrated Approach: This approach is the most challenging because the adaptation strategy is decided jointly by all the layers.

The above cross-layer approaches exhibit different advantages and drawbacks for wireless multimedia transmission, and the best solution depends on the application requirements, used protocols, algorithms at the various layers, complexity and limitations. To summarize the above mentioned approaches, we can say that the most appealing approach is the integrated approach. However, this approach is difficult to be implemented due to the increased complexity as a direct result of the big number of possible strategies and the associated parameters involved.

\section{Expected Result}

In the first part of this paper we have presented different effects of SNR on link quality. First, the throughput and the SNR are directly correlated to the distance between the sender and the receiver. Second, the through- put is directly correlated to the SNR. A good link quality is defined by a good SNR. The SNR is a good indicator of the quality of service of the link. But it is more convenient to aggregate all SNR information's available on each link into one metric indicator for each path. (We chose this new metric to find the best available path and to globally improve the network performance. Each link of the best path will have a low Bit.

Error Rate and the selected path has the best available throughput between the sender and the receiver. The measuring method of SNR helps us to have a good overview over the time of the SNR information and not just the SNR at a given selected time. It helps us to determine which link has a good stability over the time and which link has the lower probability to shut down.

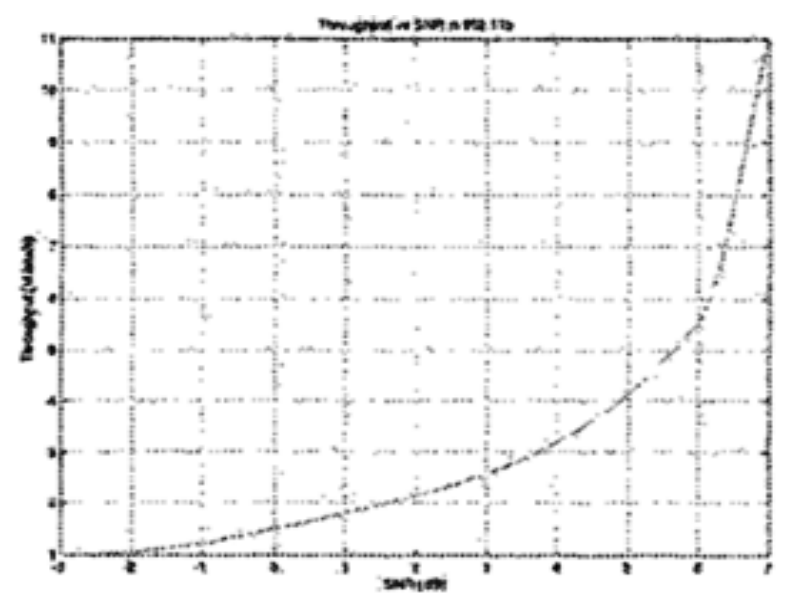

Throughput vs SNR

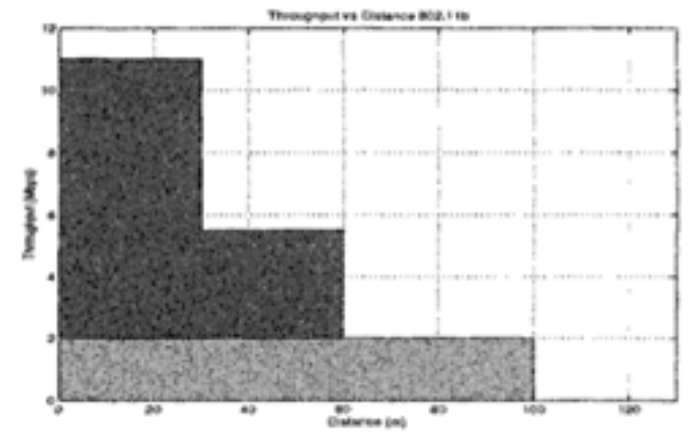

$802.11 \mathrm{~b}$

Distance vs Throughput in 


\section{Simulation Results}

NS2 simulations are made to demonstrate the proposed CLD approach in wireless networks. The results are presented here.

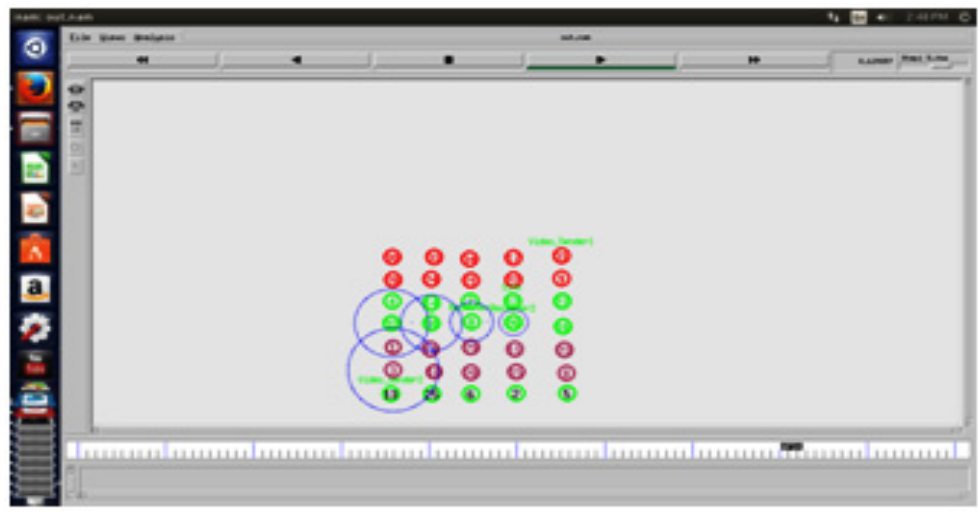

Figure 1. Shows Simulation with Wireless Network

As shown in Figure 1, the wireless network is presented using NS2 simulations. There are nodes designated as video sender and video receiver with many intermediate nodes.

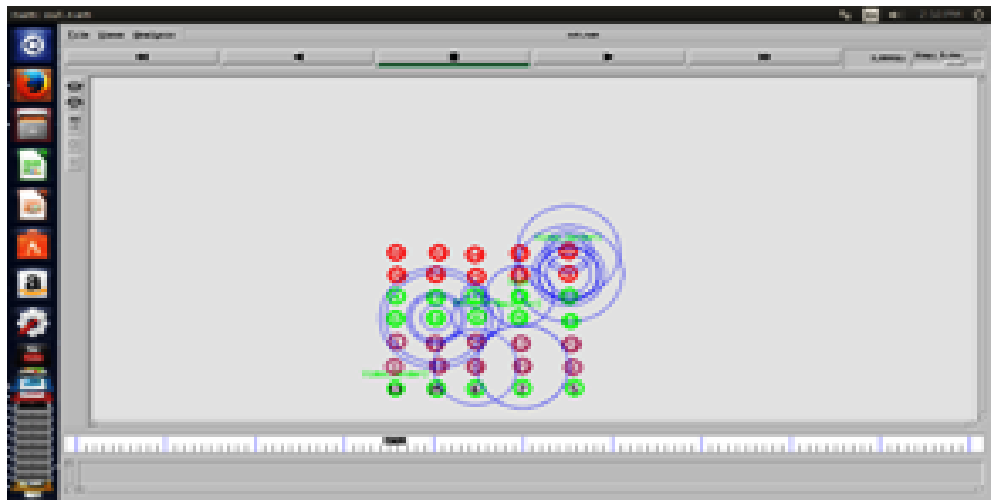

Figure 2. Simulation with Protocol Propagation

As shown in Figure 2, it is evident that the simulation shows protocol propagation among the nodes in the network.

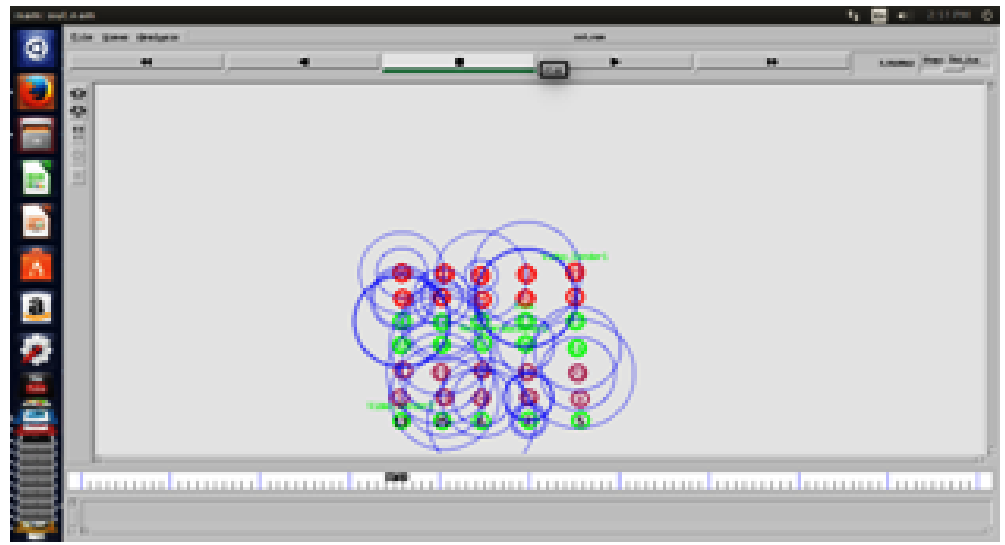

Figure 3. Data Transmission Simulation 
As shown in Figure 3, data transmission with cross layer design approach is simulated between sender and receiver.

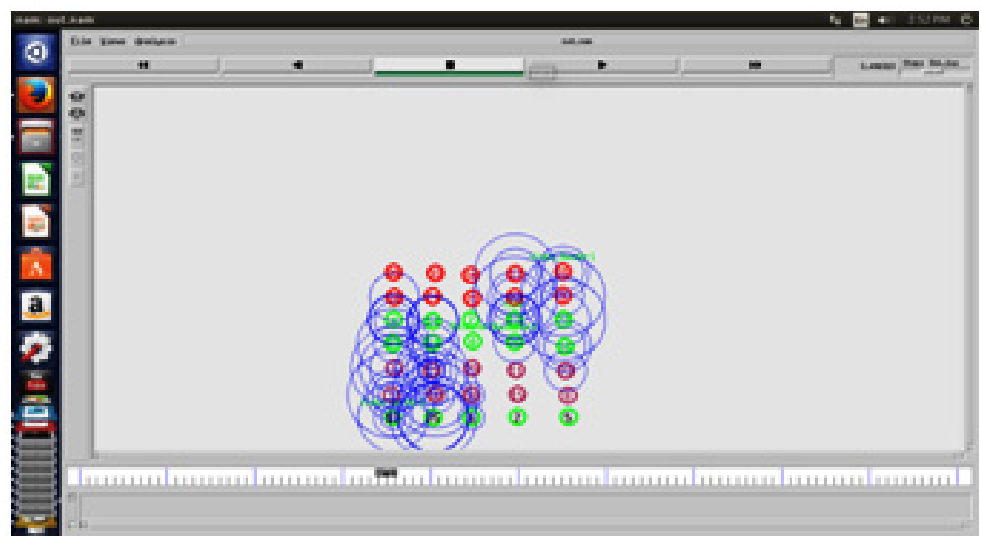

Figure 4. Data Transmission Simulation Continued

As shown in Figure 4, data transmission with cross layer design approach is simulated between sender and receiver.

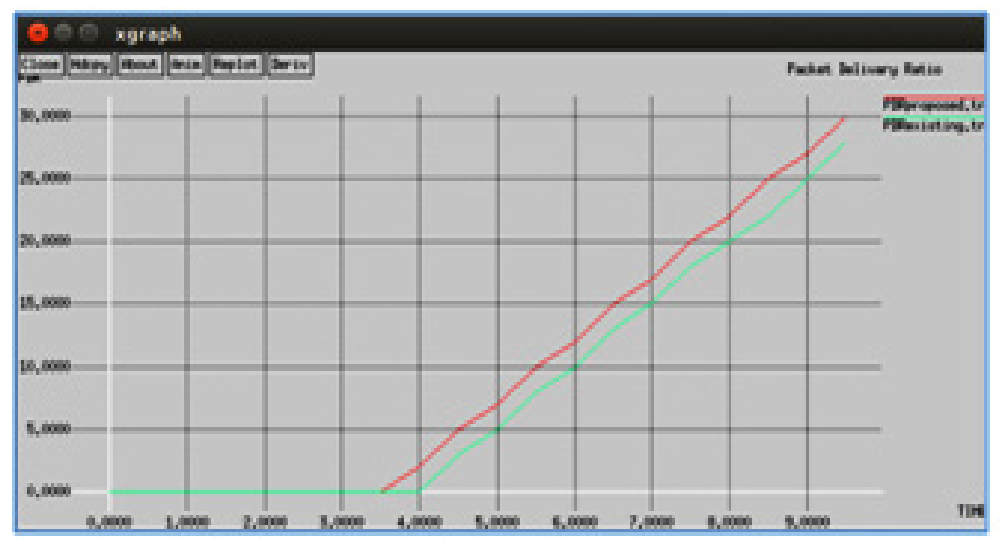

Figure 5. Packet Delivery Ratio

As shown in Figure 5, the packet delivery ratio performance of the system is presented.

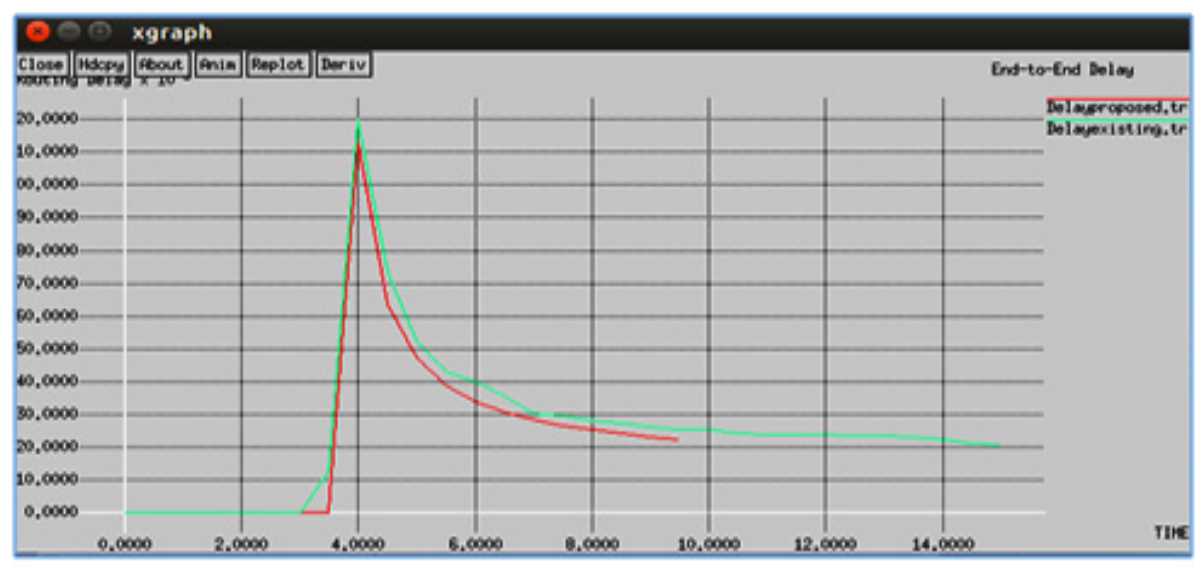

Figure 6. End to end delay Performance 
The end to end delay performance is presented in Figure 6. The results revealed that the delay is more at certain time but later it is decreased gradually and significantly.

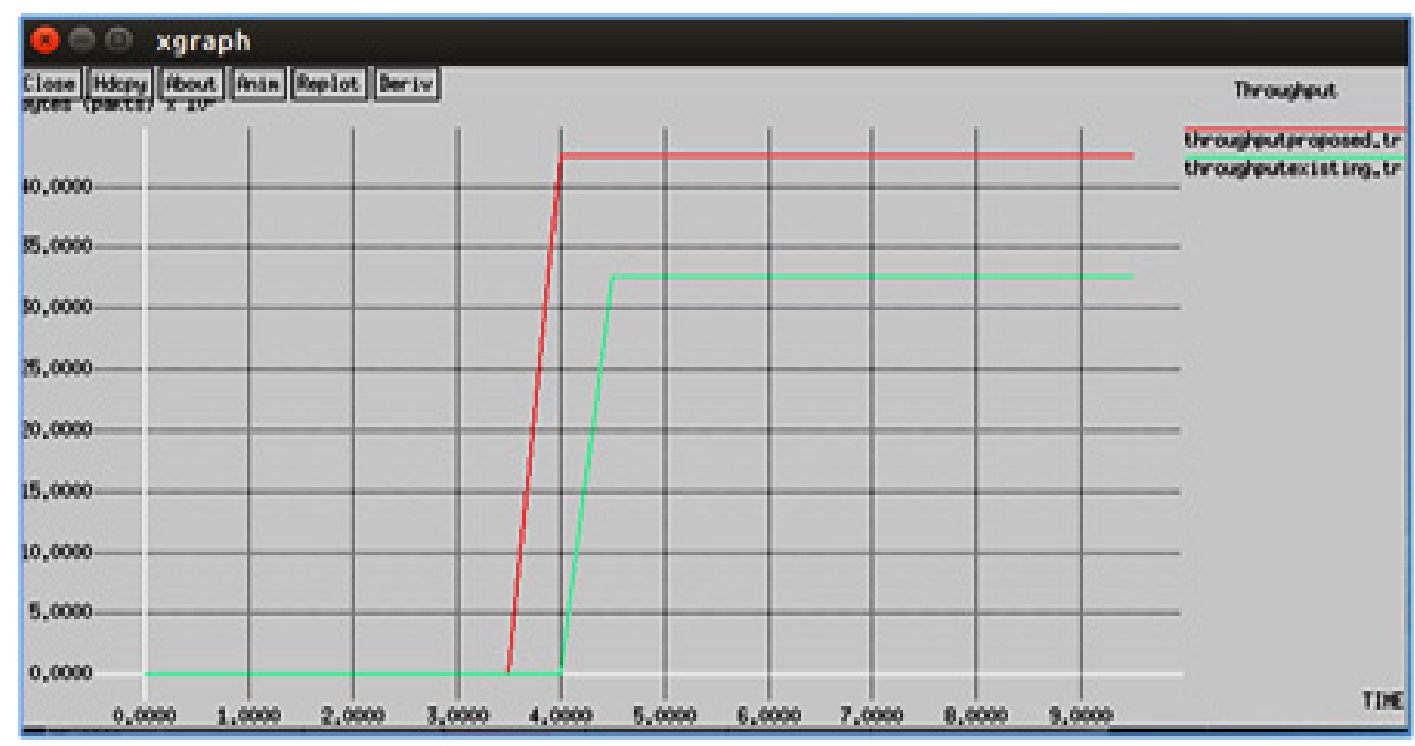

Figure 7. Throughput Performance

As can be seen in Figure 7, the throughput performance is presented. The throughput of the proposed system is high as simulation time is increased.

\section{Conclusion}

This chapter presents the challenges in designing and implementing cross layer adaptation schemes for multimedia transmission over wired and wireless networks. Cross layer adaptation for multimedia transmission will have important impact both in the research community and in the industry. More particularly, cross layer adaptation will allow better access to media content for users in a variety of locations, contexts and mobility scenarios.

\section{References}

[1] Ahmed, T. (2005). Adaptive packet video streaming over IP networks: A cross-layer approach, IEEE Journal on Selected areas in Communications, 23 (2).

[2] Blake, S. (1998). An Architecture for Differentiated Services, IETF RFC 2475.

[3] Carneiro, G., Ruela, J., Ricardo, M. (2004). Cross-layer design in 4G wireless terminals, IEEE Wireless Communications, 11 (2) p. 7- 13.

[4] Chen, J., Tiejun L., Zheng, H. (2004). Cross-layer design for QoS wireless communications, Proceedings of the 2004 International Symposium on Circuits and Systems, 2004. ISCAS '04, 2, p. II- 217-20 Vol (2).

[5] Chen J., Zheng, T. (2004). Joint cross-layer design for wireless QoS content delivery, IEEE International Conference on Communications, V. 7, p. 4243-4247.

[6] Choudhury, S., Gibson, J. (2006). Joint PHY/MAC Based Link Adaptation for Wireless LANs with Multipath Fading, IEEE Wireless Communications and Networking Conference, Vol 2, p. 757-762.

[7] Dimic, G., Sidiropoulos, N. D., Zhang, R. (2004). Medium Access Control — Physical Cross-Layer Design, IEEE Sig. Proc., vol. $21(5) 40-50$. 